\title{
Impact of Considering the ITU-R Two Slope Propagation Model in the System Capacity Trade-off for LTE-A HetNets with Small cells
}

\author{
Sofia Sousa ${ }^{*(1)}$, Fernando J. Velez ${ }^{(1)}$, and Jon M. Peha ${ }^{(2)}$ \\ (1) Instituto das Telecomunicações -DEM Universidade da Beira Interior, Faculdade de Engenharia 6201-001 Covilhã, \\ Portugalsofia.sousa@1x.it.pt,fjv@ubi.pt \\ (2) Dept. of Electrical and Computer Engineering, Dept. of Engineering \& Public Policy Carnegie Mellon University \\ Pittsburgh, PA 15213-3890, USA peha@cmu.edu
}

\begin{abstract}
This work aims at understanding and evaluating the impact of using different path loss models in the optimization trade-off of small cell (SC) networks. In LTE-A, the more realistic propagation models are the more efficient the radio and network optimization becomes. In this work we compare four urban path loss models: the urban/vehicular and pedestrian test environment from the ITU-R M. 1255 Report as well as the two slope Micro Urban Line-of-Sight (LoS) and Non-Line-of-Sight (NLoS) from the ITU-R 2135 Report. The two-slope model considers the existence of a breakpoint in the behaviour of the path loss and yields a significantly lower throughput per square $\mathrm{km}$ than a traditional one-slope model if and only if cell radius is small (coverage distances, $R$, up to breakpoint distance divided by the reuse pattern).
\end{abstract}

\section{Introduction}

In recent years, several propagation path loss models have been developed and proposed for cellular systems operating in different environments (outdoor, urban, suburban, rural, and indoor). The path loss model represents the reduction of the signal when it is propagating from the transmitter to the receiver, i.e., between the base station and mobile user. There are three different ways to model the path loss, as follows: deterministic, stochastic and empirical [1]. The deterministic model considers a specific transmitter location, a receiver location, and the properties of the environment. This type of model uses the electromagnetic wave propagation and requires the 3-D map of the propagation environment. In many cases, it is not possible to consider such a specific environment, and the appropriate approach is to consider channels that model the "typical", "worst case" or "best case" [2]. One example of the deterministic model is a ray tracing model. The stochastic models represent the environment as a series of random variables, therefore requiring less information about the environment and the use of less processing power. An empirical model is based on observation and measurements. The classification of empirical models can be further divided into time dispersive and non-time dispersive. Time dispersive provides information about time dispersive characteristics of the channel, i.e., the multipath delay spread of the channel. Non-time dispersive consider various parameters, such as distance, antenna heights, frequency and transmitter power to predict average path loss. In this work we compare the ITU-R 2135 model [3], applied to the Urban micro scenario, Line-of-Sight or Non-Line-of-Sight, and the Urban/Vehicular and Pedestrian model, defined in the ITU-R M.1255 Report [4] applied to small cells, operating at $2.6 \mathrm{GHz}$.

The remaining of the paper is organized as follows. Section II gives an overview of the considered propagation models and their application to the analysis of the frequency reuse trade-off. Section III compares the achievable supported cell physical throughput for different reuse patterns and propagation models. Finally, the main lesson learned are discussed in Section IV.

\section{Overview of the propagation models}

ITU-R was responsible for defining a global standard for the fourth generation of mobile communication systems known as international mobile telecommunications (IMT) - Advanced [5]. The ITU-R M 1225 Report has provided guidelines for evaluating a number of test environments. The scenarios under study are the outdoor-toindoor/pedestrian test environments and vehicular test environment. Outdoor-to-indoor and pedestrian test environments is characterized by small cells and low transmitter power. Base stations with low antenna heights are located outdoors; pedestrian users are located on streets and inside buildings and residences. In turn, vehicular test environment is characterized by larger cells and higher transmitter power. The path loss model defined by ITU-R M.2135-1 Report proposes models that represent the channel behaviour through deterministic category. The deterministic category encompasses all models that describe the propagation channel for a specific transmitter location, receiver location and location. For evaluation of the IMT-Advanced candidates, the ITU-R WP D defines several test environments [3]. The scenario evaluated in this work considers the microcellular scenario. The microcellular test environment focuses on small cells and high user densities and traffic loads in city centres and dense urban areas. The key characteristics of this test environment are high traffic loads, outdoor and outdoor-toindoor coverage. The channel model for urban macro-cell scenario is called urban micro (UMi). 


\subsection{Characterization of the Propagation Models}

The propagation characteristics for the outdoor-to-indoor/ pedestrian $(\boldsymbol{P e d})$ test environment are characterized by the following path loss model:

$$
P_{L P e d}=40 \cdot \log _{10}(d[\mathrm{~km}])+30 \cdot \log 10(f[\mathrm{MHz}])+49
$$

where $d$ is the separation between the mobile station and base station, in $\mathrm{km}$, and $f$ is the carrier frequency, in $\mathrm{GHz}$. Path loss model for vehicular/urban (Urb) test environment is characterized by the following path loss model:

$$
\begin{gathered}
P_{L \text { Urb }}=40 \cdot\left(1-4 \times 10^{-3} h_{B S[\mathrm{~m}]}\right) \cdot \log _{10}\left(d_{[\mathrm{km}]}\right)- \\
18 \cdot \log 10 \cdot\left(h_{B S[\mathrm{~m}]}\right)+21 \cdot \log 10\left(f_{[\mathrm{MHz}]}\right)+80
\end{gathered}
$$

where $h_{B S}$ is the base station antenna height (in $\mathrm{m}$ ), measured from the average rooftop level. For $f=2.6 \mathrm{GHz}$ and $h_{B S}=10 \mathrm{~m}$, the path loss, in $\mathrm{dB}$, is given by:

$$
\begin{aligned}
& P_{L P e d}(f=2.6 \mathrm{GHz})=40 \cdot \log 10\left(d_{[\mathrm{km}]}\right)+151.4492 \\
& P_{L \text { Urb }}(f=2.6 \mathrm{GHz})=38.40 \cdot \log 10\left(d_{[\mathrm{km}]}\right)+133.71
\end{aligned}
$$

The UMi scenario is characterized by the following path loss model. For the Outdoor scenario, there is a two-slope characteristic:

$$
\begin{gathered}
P_{\text {L UMi LoS }}=22 \cdot \log _{10}\left(d_{[\mathrm{m}]}\right)+28.0+20 \\
\log _{10}\left(f_{C[\mathrm{~Hz}]}\right), d<d_{B P} \\
P_{L \text { UMi LoS }}=40 \cdot \log _{10}\left(d_{[\mathrm{m}]}\right)+7.8-18 \cdot \log _{10}\left(h_{B S}^{\prime}\right) \\
-18 \cdot \log _{10}\left(h^{\prime}{ }_{U T}\right)+2 \cdot \log _{10}\left(f_{C[H z]}\right), d>d_{B P} \\
P_{\text {L UMi NLoS }}=36.7 \cdot \log _{10}(d)+22.7+26 \cdot \log _{10}\left(f_{c}\right)
\end{gathered}
$$

where $h_{B S}=10 \mathrm{~m}$ and the considered street width is $20 \mathrm{~m}$, while the average building height is $20 \mathrm{~m}$. Variables $h_{B S[\mathrm{~m}]}=h_{B S}-1$ and $h^{\prime}{ }_{U T[\mathrm{~m}]}=h_{U T}-1$ also stand. The breakpoint distance, $d_{B P}$, is calculated by:

$$
d_{B P}=4 \cdot h_{B S}^{\prime} \cdot h_{U T}^{\prime} \cdot f_{C} / c
$$

where $f_{\mathrm{c}}$ is the centre frequency, in Hertz, $c=3.0 \times 10^{8} \mathrm{~m} / \mathrm{s}$ is the propagation velocity in free space. Therefore, one obtains $d_{B P}$ UMi LoS $=156 \mathrm{~m}$. By considering these assumptions, the path loss, in $\mathrm{dB}$, is given by:

$$
\begin{aligned}
& P_{\text {L UMi Los }}(d)=22 \cdot \log _{10}\left(d_{[\mathrm{m}]}\right)+36.29947, d<156 \mathrm{~m} \\
& P_{\text {L UMi Los }}(d)=40 \cdot \log _{10}\left(d_{[\mathrm{m}]}\right)-3.12788, d \geq 156 \mathrm{~m} \\
& P_{\text {L UMi NLoS }}(d)=36.7 \cdot \log _{10}\left(d_{[\mathrm{m}]}\right)+33.48
\end{aligned}
$$

The noise power at the receiver is calculated by:

$$
N[d B m]=-174+10 \cdot \log _{10}(B W[\mathrm{~Hz}])+N_{f}[d B]
$$

where $B W$ is the bandwidth of the radio channel being used and $N_{f}$ is the noise figure at the receiver. The assumed transmitter power and gains are $P_{t}=-7 \mathrm{dBW}, G_{t}=17 \mathrm{dBi}$, $G_{r}=0 \mathrm{dBi}$. The following parameters are also considered: $B W=10 \mathrm{MHz}, N_{f}=5 \mathrm{~dB}$ and $f=2.6 \mathrm{GHz}$.

\section{Cellular System}

In this section we describe a cellular system, and how to make coverage planning and frequency allocation, in terms of how to calculate the interference, carrier-to- interference ratio $(C / I)$ and carrier-to-interference-plus-noise ratio $(C N I R)$ in an OFDM system with static allocation scheme or fixed channel allocation. We consider a symmetrical hexagonal cell plan, a modulation scheme requiring a minimum CNIR and the transmitters use a constant transmitter power, $P_{t}$. Coverage planning is necessary to give mobile users a guarantee of the quality of the received signal for both the downlink (DL) and uplink (UL). One of the objectives is to design a wireless network where for a given available bandwidth, the system achieves the highest capacity possible. We address the downlink of the system for the worst-case situation, where the UE is at the cell edge, where the channel is used in all these co-channel cells.

In a fully symmetrical hexagonal plan with a given frequency reuse pattern $K$, we consider the reuse distance, $D$, is $D=\sqrt{3 k} R$, where $R$ is the radius of the hexagonal cell. The possible values for $K$ are $K=1,3,4$, 7, where $K=1$ is the case where all channels are used in all cells. As, for the very short coverage distances associated with small cells, the approximate $C / I$ formulation considered in the previous works [6] has shown to be inadequate, a more detailed approach is sought in this work.

The $C / I$ ratio formulation used in the previous work is given by the following equation:

$$
\frac{C}{I}=\frac{1}{2\left(\mathrm{r}_{\mathrm{cc}}+1\right)^{-\gamma}+2 \mathrm{r}_{\mathrm{cc}}{ }^{-\gamma}+2\left(\mathrm{r}_{\mathrm{cc}}-1\right)^{-\gamma}} \approx \frac{\mathrm{r}_{\mathrm{cc}}{ }^{\gamma}}{6}
$$

where $r_{c c}$ is the co-channel reuse factor, given by $r_{c c}=D / R$. Instead, we have obtained a more detailed equation that represents $C / I$ with exact values for all the reuse distances, from the eNBs of the first, second and third tiers of cochannel cells (interferers) to the UE. Considering the first three tiers of interferers is a valid approximation, since the interference obtained from the second and third tier is negligible. In these equations we consider the exact position of each interferer, in each tier of interferers, in opposition to the equations with values for the reuse distances. With hexagonal cell topologies for the macroand pico- cellular layers, in the DL, for $K=3, C / I$ is given by the following equations for the $1^{\text {st }}, 2^{\text {nd }}$ and $3^{\text {rd }}$ rings:

$$
\begin{aligned}
& \quad \frac{C}{I_{1} t}=\frac{R^{-\gamma}}{2(D+0.66394 R)^{-\gamma}+2(D-0.31395 R)^{-\gamma}+(D+R)^{-\gamma}+(D-R)^{-\gamma}} \\
& {\frac{C}{2^{n d}}}_{2^{n d}}=\frac{R^{-\gamma}}{2(\sqrt{3} D+0.88915 R)^{-\gamma}+2(\sqrt{3} D+0.08591 R)^{-\gamma}+2(\sqrt{3} D-0.84799 R)^{-\gamma}} \\
& \frac{C}{I_{3}{ }^{r d}}=\frac{R^{-\gamma}}{2(2 \mathrm{D}+0.55802 R)^{-\gamma}+2(2 \mathrm{D}-0.47727 R)^{-\gamma}+(2 \mathrm{D}+R)^{-\gamma}+(2 \mathrm{D}-R)^{-\gamma}}
\end{aligned}
$$

By considering the above formulation, Figures 1 and 2 analyse the variation of the CNIR, with the distance $d$ from the cell centre to the UE within a cell, where $0 \leq d \leq R$, for cell coverage radii $R=30$ and $300 \mathrm{~m}$. The CNIRs obtained for UMi NLoS, Urban and Pedestrian are similar, as shown in Figure 2, since the respective propagation exponents are $\gamma=3.67,3.84$ and 4 .

However, in the UMi LoS scenario, for short coverage distances, as shown in Figure 1, since the propagation exponent is $\gamma=2.2$, the CNIR is considerably lower. For $R \mathrm{~s}$ longer than $R_{o}$, where $R_{o}=d_{B P} / K$ for UMi LoS, the obtained CNIR is gradually closer to the rest of the path loss curves, as for $R \mathrm{~s}$ longer than $d_{B P}$, since the propagation exponent of UMi LoS is $\gamma=4$, the obtained CNIR is higher than the rest of the path loss models curves, as shown in Figure 2. 


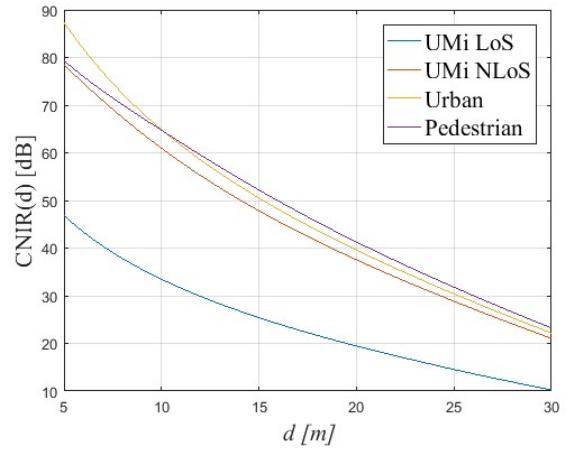

Figure 1. Comparison of CNIR between the UMi LoS, UMi NLoS, Urban and Pedestrian propagation models, for $K=3$ and $R=30 \mathrm{~m}$.

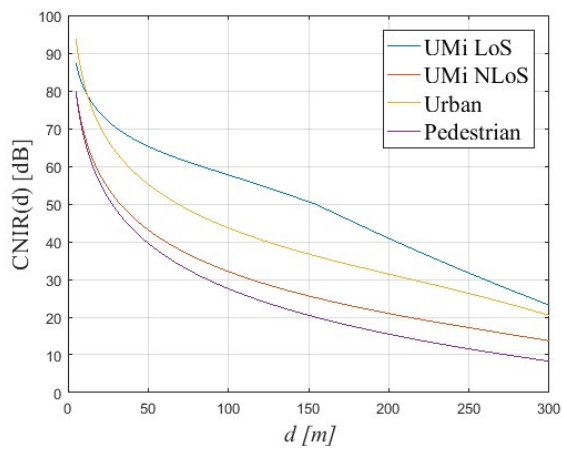

Figure 2. Comparison of CNIR between the UMi LoS, UMi NLoS, Urban and Pedestrian propagation models, for $K=3$ and $R=300 \mathrm{~m}$.

\section{Supported Cell/Sector Physical Throughput}

The LTE-A system capacity is analysed by following the formulation from [7] for an implicit function procedure to compute the supported cell physical throughput, $R_{b-s u p}$. This analysis considers different values of the reuse pattern, e.g., $K=1$. To map $C N I R_{\min }$ into the supported throughput, $R_{b}$, we have used the values for $C N I R_{\min }$ from [8]. By extrapolating the gathered information, it is possible to map the CNIR into MCS index, Modulation Order Transport Block Size (ITBS) index and TBS.

Figure 3 shows the results for the supported throughput per cell, $R_{b-\text { sup }}$ for $K=1$. Although the curves for $K=3$ are not presented, we observe that for cells with $R$ s shorter than $125 \mathrm{~m}$ (for $K=1)$ and $R \mathrm{~s}$ shorter than $70 \mathrm{~m}(K=3)$, more optimistic results are obtained with the Pedestrian path loss model, followed by the Urban, UMi NLoS and UMi LoS models. This means that the UMi LoS model presents the most pessimistic results for small cell coverage ranges. Nevertheless, for longer cell ranges, for example $R \geq 225$ $\mathrm{m}(K=1)$ and $R \geq 150 \mathrm{~m}(K=3)$, the best results are obtained for UMi LoS, followed by Urban, UMi NLoS. The worst results are obtained for the Pedestrian Path Loss model. To understand the impact of considering a more realistic propagation model that considers the existence of a breakpoint in the behaviour of the path loss, in radio and network optimization, we analyse the supported throughput per unit area, $R_{b-u a}$, in Figure 4.

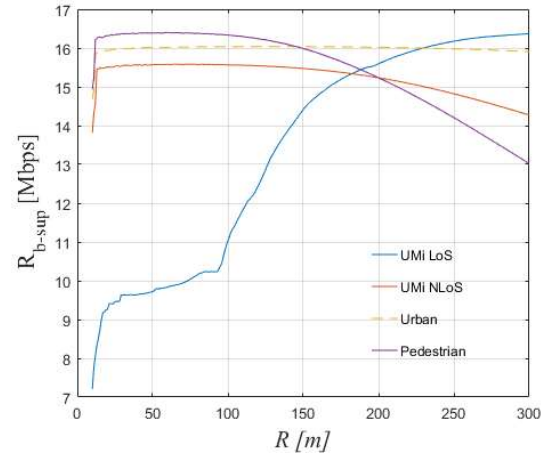

Figure 3. Comparison of the equivalent supported throughput between the UMi LoS, UMi NLoS, Urban and Pedestrian path loss models, $K=1, B W=10 \mathrm{MHz}$

$R_{b-u a}$ is obtained by multiplying the number of cells per unit area by the supported throughput for $K$. The reduction of the supported throughput while considering the UMi LoS propagation, $R_{b-u a \_U M i}$ Los, is compared to the supported throughput while considering Pedestrian propagation scenario, $R_{b-u a_{-} P e d}$, is defined as $\operatorname{Red}_{R b-u a}$ and is obtained by the ratio defined in the following equation:

$\operatorname{Red}_{R b-u a}[\%]=\frac{R_{b-u a_{-} U M i_{-} L o S}-R_{b-u a_{-} P e d}}{R_{b-u a} \text { Ped }} \cdot 100(17)$.

For $K=1$, we observe in Figures 4 and 5 that, for cells with short coverage distances, for example, $R=40 \mathrm{~m}$, the supported throughput per unit area, $R_{b-u a}$, obtained with two-slope model (UMi LoS) is reduced by $41.12 \%$ compared to the results that arise from applying the single slope model (Pedestrian scenario), as shown in Figure 10. For $K=3$, the two-slope model has reduction of $37.23 \%$ in $R_{b-u a}$ compared to the values obtained with single slope model. Figure 5 shows the ratio (percentage) between the $R_{b-u a}$ for the two-slope model (UMi LoS) divided by the one slope model (Pedestrian). The results with two-slope model overcomes the value obtained for $R_{b \text {-ua }}$ from the one slope model for coverage distances longer than $R=180 \mathrm{~m}$ and $R$ $=96 \mathrm{~m}$, for $K=1$ and 3 , respectively. In fact, values of Red $R_{b-u a}$ higher than zero means a reduction of the throughput when considering the two-slope model, whereas negative values (obtained for $R$ s longer than these values) mean that the single-slope modes are more pessimistic in the determination of the supported throughput per unit area).

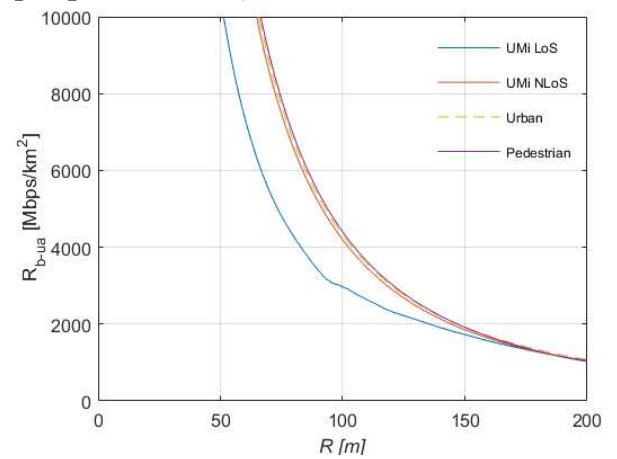

Figure 4. Comparison of the equivalent supported throughput per unit area between the UMi LoS, UMi NLoS, Urban and Pedestrian path loss models, $K=1$, $B W=10 \mathrm{MHz}$ 


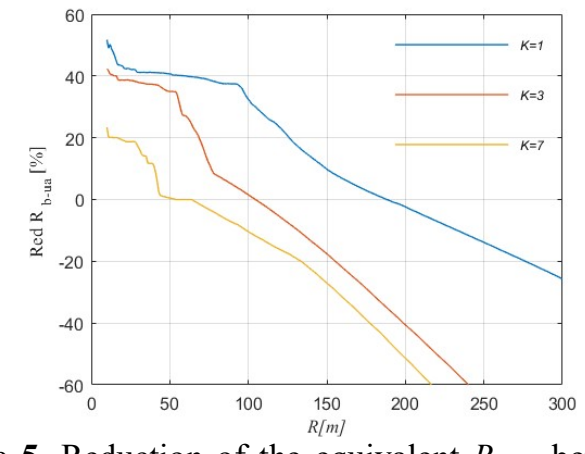

Figure 5. Reduction of the equivalent $R_{b-\text { - } a}$ between the UMi LoS and Pedestrian path loss models in percentage, for $K=1,3$ and $7, B W=10 \mathrm{MHz}$

From this analysis, we have learned that, as the two-slope model captures the actual behaviour of the propagation in small cell environments, whose breakpoint defines the change of the propagation characteristics, by considering the ITU-R 2135 UMi LoS propagation model, a more realistic characterization of the CNIR and supported throughput trade-off is achieved, where lower values of the throughput per unit area are achievable for shorter $R$ s while for longer $R \mathrm{~s}$ the consideration of the two-slope model leads to higher values of system capacity.

\section{Conclusions}

This work has evaluated the impact of considering different path loss models in the optimization of the frequency reuse and system capacity trade-offs of small cell networks. Also we have obtained a more detailed equation that represents carrier-to-interference ratio, $C / I$, with exact values for all the reuse distances, from the eNBs of the first, second and third tiers of co-channel cells (interferers) to the UE.

We have learned from the analysis that by considering the realistic assumptions from the ITU-R two-slope, for coverage distances, $R$, up to breakpoint distance divided by $K, d_{B P} / K, R_{b \text {-sup }}$ is much lower than expected when traditional single-slope models are assumed. For $R$ s longer than $d_{B P} / K$ the results for $R_{b \text {-sup }}$ are increasing with $R$, whereas they are steady or decreasing with $R$ while considering the traditional single-slope propagation models. This increase is due to the existence of a low propagation exponent (slope) in term of coverage and a high slope in terms of interference for $d_{B P} / K \leq R \leq d_{B P}$. Recent research has found that a two-slope propagation model is more accurate than the traditional one-slope models [3]. We find that these two models yield similar results if cell radius is large compared to the break-point of the two-slope model divided by reuse pattern. However, when cell radius is small, throughput achievable with a two-slope model is significantly lower. We observed a throughput per area that is 20 to $45 \%$ lower in the scenarios considered. This difference in throughput exists because the one-slope model uses a higher propagation exponent for devices that are closer to the transmitter. Thus, as cellular carriers reduce cell size to support growing traffic volume, use of traditional propagation models may produce designs with inadequate capacity. Moreover, assuming that the two-slope model is correct, these results also show that the gains in capacity per area from reducing cell size get smaller when cell radius falls below this threshold. This means that operators may find it more costeffective at that point to meet their growing capacity needs by decreasing the frequency reuse factor or increasing spectrum holdings rather than decreasing cell size, assuming that spectrum is obtainable or that frequency reuse is not already at its minimum.

\section{Acknowledgements}

This work has been partially supported and funded by CREaTION, COST CA 15104, ECOOP, UID/EEA/50008/ 2013, SFRH/BSAB/113798/2015, 3221/BMOB/16 Carnegie Mellon University Portugal Faculty Exchange Programme grant, EFATraS, Bolsa BID/ICI-FE/Santander Universidades-UBI/2016 and ORCIP.

\section{References}

1. V. S Abhayawardhana et al. "Comparison of empirical propagation path loss models for fixed wireless access systems," in Proc.of IEEE 61st Vehicular Technology Conference. vol. 1. IEEE, 2005.

2. IEEE $802.16 \mathrm{~m}-08 / 004 \mathrm{r} 5$, IEEE $802.16 \mathrm{~m}$ Evaluation Methodology Document, January 2009.

3. Guidelines for evaluation of radio interface technologies for IMT-Advanced, Report ITU-R M.2135-1, Dec. 2009. (https://www.itu.int/dms pub/itu-r/opb/rep/RREP-M.2135-1-2009-PDF-E.pdf)

4. Guidelines for evaluation of radio transmission technologies for IMT-2000, Report ITU-R M.1255, 1997. (https://www.itu.int/dms pubrec/itu-r/rec/m/R-RECM.1225-0-199702-I!!PDF-E.pdf)

5. LTE - Advanced A Practical Systems Approach to Understanding 3GPP LTE Releases 10 and 11 Radio Access Technologies, Academic Press, 2014.

6. Fernando J. Velez et al., "Basic Limits for LTEAdvanced Radio and HetNet Optimization in the Outdoorto-indoor Scenario", in Proc.of IEEE BlackSeaCom 2016, Varna, Bulgaria, June 2016.

7. Daniel Robalo and Fernando J. Velez, "Economic trade-off in the optimization of carrier aggregation with enhanced multi-band scheduling in LTE-Advanced scenarios," EURASIP Journal on Wireless Communications and Networking, vol. 2015, no. 1, 2015, pp. 189 (doi: 10.1186/s13638-015-0371-9).

8. 3GPP, TS 36.212, V11.3.0. Technical Specification Group Radio Access Network; Evolved Universal Terrestrial Radio Access (E-UTRA); Multiplexing and channel coding, 3GPP Std., June 2013. 